\section{Immuntherapie bei fortgeschrittenem NSCLC}

\author{
Auch Patienten mit nichtkleinzelligem Lungenkarzinom (NSCLC) profitieren \\ von immuntherapeutisch wirksamen Medikamenten. Die Evidenz für die \\ Checkpointinhibition mit Pembrolizumab scheint in großen Studien wie \\ KEYNOTE-010 überzeugend.
}

$\mathrm{D}$ ie Behandlung von Patienten mit NSCLC hat sich durch die Therapie mit zielgerichteten Molekülen deutlich verbessert. Einen weiteren Innovationsschub brachten immunmodulatorische Wirkprinzipien, die z.B. den $\mathrm{PD}($,programmed cell death protein")-1-Signalweg blockieren. $\mathrm{Zu}$ dieser Substanzgruppe gehört Pembrolizumab. Aufgrund positiver Behandlungsergebnisse bei Patienten mit metastasiertem NSCLC mit positivem PD-L1(PD-Ligand 1)-Status und Refraktärität gegenüber Platin erhielt Pembrolizumab in den USA schon eine Zulassung. Bestätigt werden sollten diese Ergebnisse mit der Phase-II/III-Studie KEYNOTE-010, an der 1.034 prospektiv erfasste, PD-L1-positive NSCLC-
Patienten nach Progress unter platinbasierter Chemotherapie teilnahmen.

In der Gesamtpopulation betrug das mediane Gesamtüberleben unter Pembrolizumab 10,4 Monate (Dosierung 1: $2 \mathrm{mg} / \mathrm{kg}$; $\mathrm{q} 3 \mathrm{w}$ ) und 12,7 Monate (Dosierung 2: $10 \mathrm{mg} / \mathrm{kg} ; \mathrm{q} 3 \mathrm{w}$ ) im Vergleich zu 8,5 Monaten unter der Chemotherapie mit Docetaxel $\left(75 \mathrm{mg} / \mathrm{m}^{2}\right.$; $\left.\mathrm{q} 3 \mathrm{w}\right)$. Der Unterschied zwischen beiden Immuntherapie-Dosierungen und Docetaxel war signifikant ( $\mathrm{p}=0,0008$ bzw. $\mathrm{p}<0,0001$ ). Beim medianen progressionsfreien Überleben (PFS) zeigte sich kein Unterschied. Patienten, in deren Tumoren mindestens $50 \%$ der Zellen PD-L1 exprimierten, überlebten im Vergleich zu den mit Docetaxel behandelten Patienten deutlich länger: Unter der niedrigeren Pembrolizumab-Dosis median 14,9 versus 8,2 Monate (Hazard Ratio [HR] 0,54; $p=0,004)$, unter der höheren 17,3 versus 8,2 Monate (HR 0,50; $p<0,0001$ ). Auch das PFS war in dieser Subgruppe unter der Immuntherapie jeweils signifikant verlängert. Nebenwirkungen vom Grad 3-5 waren unter den beiden AntikörperDosierungen mit 13 bzw. $16 \%$ seltener als unter Docetaxel (35\%).

Fazit: Pembrolizumab verlängert das Gesamtüberleben von chemotherapeutisch vorbehandelten Patienten mit fortgeschrittenem NSCLC und PD-L1-Positivität. Der Nachweis von PD-L1-Molekülen auf den Tumorzellen scheint als Biomarker für die Auswahl von NSCLCPatienten geeignet, die von der PD-1-Inhibition profitieren können.

Barbara Kreutzkamp

Herbst RS et al. Pembrolizumab versus docetaxel for previously treated, PD-L1-positive, advanced non-small-cell lung cancer (KEYNOTE-010): a randomised controlled trial. Lancet. 2016;387(10027):1540-50.

\title{
Lokal fortgeschrittenes Nichtplattenepithel- NSCLC: Pemetrexed ohne Vorteile
}

\author{
Welchen Nutzen hat das Regime \\ Pemetrexed/Cisplatin mit anschlie- \\ ßender Pemetrexed-Konsolidierung \\ bei Patienten mit nicht resektablem, \\ lokal fortgeschrittenem Nichtplat- \\ tenepithelkarzinom der Lunge? Mit \\ der PROCLAIM-Studie ging man \\ dieser Frage nach.
}

$\mathrm{D}$ er Standard für Patienten mit inoperablem nichtkleinzelligem Lungenkarzinom (NSCLC) im Stadium III besteht aus einer platinbasierten Doublette plus thorakaler Strahlentherapie (TRT). Exakte Substanz- und Dosisempfehlungen für die Radiochemotherapie fehlen. Etabliert haben sich u. a. Etoposid/Cisplatin oder Carboplatin/Paclitaxel mit RTDosierungen von 60-66 Gy. In der PhaseIII-Studie PROCLAIM wurde die Effektivität von Pemetrexed als Kombinations- partner geprüft. Pemetrexed ist beim NSCLC aktiv, erhöht in vitro die RT-Sensitivität und ist relativ gut verträglich. Patienten mit nichtresektablem NSCLC im Stadium IIIA/B erhielten randomisiert Pemetrexed plus Cisplatin (Gruppe A) oder den Standard Etoposid/Cisplatin (Gruppe B) plus jeweils eine StandardTRT. Für die Konsolidierung war in Gruppe A Pemetrexed, in Gruppe B eine platinbasierte Doublette vorgesehen.

Noch bevor der primäre Endpunkt Gesamtüberleben (OS) statistisch auswertbar war, wurde die Studie wegen absehbaren Nichterreichens einer Überlegenheit der interventionellen Therapie vorzeitig abgebrochen. Das mediane OS in Gruppe A betrug 26,8 Monate versus 25,0 in Gruppe B (Hazard Ratio [HR] 0,98, $95 \%$-Konfidenzintervall [95\%-KI] $0,79-1,20 ; p=0,831)$. Allerdings traten bei den Patienten unter Pemetrexed/Cis- platin plus Pemetrexed-Konsolidierung deutlich weniger schwere Nebenwirkungen auf (64,0 vs. $76,8 \% ; p=0,001)$. Das galt auch für Neutropenien (24,4 vs. $44,5 \%$; $<<0,001)$. Das progressionsfreie Überleben war im Trend für die Gruppe A besser. Auch schienen Patienten mit einer schlechten OS-Prognose eher von Pemetrexed zu profitieren, sodass diese Substanz auf jeden Fall weiter erforscht werden sollte.

Fazit: Bei Patienten mit nicht resezierbarem NSCLC im Stadium III ergab sich für Pemetrexed/Cisplatin plus StandardTRT mit anschließender PemetrexedKonsolidierung keine Überlegenheit beim OS im Vergleich zu einer bisherigen Standardbehandlung. Die Verträglichkeit von Pemetrexed war allerdings deutlich besser. Barbara Kreutzkamp

Senan S et al. PROCLAIM: Randomized phase III trial of pemetrexed-cisplatin or etoposide-cisplatin plus thoracic radiation therapy followed by consolidation chemotherapy in locally advanced nonsquamous non-small-cell-lung cancer. J Clin Oncol. 2016;34(9):953-62. 